\title{
TOPOLOGICALLY UNREALIZABLE AUTOMORPHISMS OF FREE GROUPS
}

\author{
JOHN R. STALLINGS ${ }^{1}$
}

\begin{abstract}
Let $\phi: F \rightarrow F$ be an automorphism of a finitely generated free group. It has been conjectured (I heard it from Peter Scott) that the fixed subgroup of $\phi$ is always finitely generated. This is known to be so if $\phi$ has finite order [1], or if $\phi$ is realizable by a homeomorphism of a compact 2-manifold with boundary [2]. Here we give examples of automorphisms $\phi$, no power of which is topologically realizable on any 2-manifold; perhaps the simplest is the automorphism of the free group of rank 3, given by $\phi(x)=y, \phi(y)=z, \phi(z)=x y$.
\end{abstract}

1. PV-matrices and automorphisms. By a PV-matrix is meant an $n \times n$ integer matrix of determinant \pm 1 , having one eigenvalue, $\lambda_{1}$, of absolute value greater than 1 , and $n-1$ eigenvalues of absolute value less than 1. The terminology "PV" is used because $\lambda_{1}$ is a Pisot-Vijayaraghavan number [3].

A PV-automorphism of a free abelian group of rank $n$ is an automorphism whose matrix is a PV-matrix, (PV-ness is independent of the basis). A PV-automorphism of a free (nonabelian) group, is an automorphism whose abelianization is PV.

1.1. If $M$ is an $n \times n P V$-matrix and $n \geqslant 3$, then, since $\left|\lambda_{1} \lambda_{2} \ldots \lambda_{n}\right|=1$, no eigenvalue $\lambda_{i}$ is the inverse of any $\lambda_{j}$.

1.2. If $M$ is a PV-matrix, then every positive integral power $M^{k}$ is a PV-matrix, since the eigenvalues of $M^{k}$ are the $k$ th powers of those of $M$.

A simple $3 \times 3$ example of a PV-matrix is

$$
\left(\begin{array}{lll}
0 & 1 & 0 \\
0 & 0 & 1 \\
1 & 1 & 0
\end{array}\right)
$$

with eigenvalues approximately

$$
\lambda_{1}=1.3247, \quad \lambda_{2}, \lambda_{3}=-0.6624 \pm 0.5623 \sqrt{-1} .
$$

Correspondingly, $\phi(x)=y, \phi(y)=z, \phi(z)=x y$ describes a PV-automorphism of the free group with basis $\{x, y, z\}$.

2. Eigenvalues of automorphisms of a 2-manifold.

2.1. Let $h: T \rightarrow T$ be an orientation-preserving homeomorphism of a closed, orientable 2-manifold onto itself. Then the eigenvalues of the homology map

$$
h_{*}: H_{1}(T) \rightarrow H_{1}(T)
$$

occur in inverse pairs; that is, they can be listed $\lambda_{1}, \lambda_{2}, \ldots, \lambda_{g}, \lambda_{1}^{-1}, \lambda_{2}^{-1}, \ldots, \lambda_{g}^{-1}$.

Received by the editors September 29, 1980.

1980 Mathematics Subject Classification. Primary 20E05.

${ }^{1}$ Partly supported by NSF grant MCS 77-04242. 
The reason is that $h_{*}$ preserves an alternating bilinear form, the intersection number, and thus $h_{*}$ is symplectic, and every symplectic matrix is similar to its inverse. (Cf. Exercise 1, §6.9, p. 377 in [4].)

2.2. Let $h: T \rightarrow T$ be an orientation-preserving homeomorphism of a compact, orientable, connected 2-manifold, with $\beta \geqslant 1$ boundary components, onto itself, such that $h$ maps each boundary component to itself. Then the eigenvalues of the homology map

$$
h_{*}: H_{1}(T) \rightarrow H_{1}(T)
$$

consist of two sorts: There are $\beta-1$ eigenvalues $=1$, and the remaining eigenvalues occur in inverse pairs.

The reason for this is that the subgroup $S$ of $H_{1}(T)$ generated by the boundary components is a free abelian direct summand of rank $\beta-1$; and $h_{*}$ maps $S$ to itself by the identity. On the quotient by $S, h_{*}$ is the homology map on the closed manifold obtained by capping off the boundary components with 2-cells, and to this we apply 2.1 .

\section{PV-automorphisms are not realizable.}

THEOREM. Let $\phi: F \rightarrow F$ be a $P V$-automorphism of a free group of infinite rank $n>3$. Then, for every integer $k>1, \phi^{k}$ is not realizable, as the automorphism on fundamental group, by any homeomorphism $h: T \rightarrow T$ of any 2-manifold $T$.

Proof. There are two cases, $T$ orientable or not.

Orientable case. If $T$ is orientable, then $h^{2}$ is orientation preserving and permutes the boundary components of $T$; this permutation has some finite order $q$, so that $h^{2 q}$ is orientation preserving and maps each boundary component to itself. The homomorphism

$$
h_{*}^{2 q}: H_{1}(T) \rightarrow H_{1}(T)
$$

is the abelianization of $\phi^{2 k q}$. This is a PV-automorphism by 1.2, and thus none of the eigenvalues of $h_{*}^{2 q}$ is the inverse of any other by 1.1. This contradicts 2.2.

Nonorientable case. If $T$ is nonorientable, let $T^{\prime} \rightarrow T$ be its orientable double cover. The homeomorphism $h: T \rightarrow T$ lifts to a homeomorphism $h^{\prime}: T^{\prime} \rightarrow T^{\prime}$. There is a transfer homomorphism

$$
\tau: H_{1}(T) \rightarrow H_{1}\left(T^{\prime}\right)
$$

such that $\tau \circ h_{*}=h_{*}^{\prime} \circ \tau$, and such that the composition

$$
H_{1}(T) \rightarrow H_{1}\left(T^{\prime}\right) \rightarrow H_{1}(T)
$$

is multiplication by 2 .

If we take the coefficient group to be the field of rational numbers-this does not change any argument on eigenvalues-then $\tau$ embeds $H_{1}(T)$ as a subspace of $H_{1}\left(T^{\prime}\right)$ which is invariant under $h_{*}^{\prime}$ and on which $h_{*}^{\prime}$ is isomorphic to $h_{*}$.

Now, as in the orientable case, there is some positive integer $q$ such that $\left(h^{\prime}\right)^{2 q}$ is orientation preserving and maps each boundary component of $T^{\prime}$ to itself. The list 
of eigenvalues of $\left(h_{*}^{\prime}\right)^{2 q}$ includes, by the transfer argument, the eigenvalues of $h_{*}^{2 q}$. The latter are eigenvalues of the abelianization of $\phi^{2 k q}$, which, as before, do not include any inverse pairs.

There are $n$ eigenvalues of $h_{*}^{2 q}$, since the rank of $H_{1}(T)$ is the rank of $F$, which is $n$. A Euler characteristic argument shows that the rank of $H_{1}\left(T^{\prime}\right)$ is $2 n-1$. Therefore, there are not enough additional eigenvalues of $\left(h_{*}^{\prime}\right)^{2 q}$ to make up a set of eigenvalues satisfying 2.2 .

\section{Comments.}

4.1. Suppose that $\phi: F \rightarrow F$ is a $\mathrm{PV}$-automorphism and that $S \subset F$ is a subgroup of finite index with $\phi(S)=S$. I suspect that $\phi \mid S$ is not realizable by a surface homeomorphism and that this can be proved by examining eigenvalues. If $S$ is a normal subgroup and $\phi$ induces the identity on $G=F / S$, then $\phi$ determines a ZG-automorphism on the abelianization of $S$. Can this automorphism have a symmetric set of eigenvalues (satisfying 2.2)? This seems to involve the question: What does the fact that $F$ is free imply about the structure of the abelianization of $S$ as a ZG-module?

4.2. Every automorphism $\phi: F \rightarrow F$ of a free group of rank $n$, whose abelianization has determinant +1 , leaves something fixed modulo the $(n+1)$ st term in the lower central series.

Proof. Define

$$
F_{1}=F, \quad F_{k+1}=\left[F, F_{k}\right] .
$$

Then the quotients of the lower central series $L_{k}=F_{k} / F_{k+1}$ form a free Lie algebra over $Z$, and $\phi$ induces automorphisms $\phi_{k}: L_{k} \rightarrow L_{k}$. Tensor with the complex numbers $C$. Then $\phi_{1}$ has eigenvalues $\lambda_{1}, \ldots, \lambda_{n}$, and corresponding eigenvectors $\xi_{1}, \ldots, \xi_{n}$ in $L_{1} \otimes \mathbf{C}$. However, we need to have $\xi_{n-1}$ and $\xi_{n}$ linearly independent, so that if $\lambda_{n-1}=\lambda_{n}$, it may be necessary to change the defining equation for $\xi_{n}$ from $\phi_{1}\left(\xi_{n}\right)=\lambda_{n} \xi_{n}$ to $\phi_{1}\left(\xi_{n}\right)=\lambda_{n} \xi_{n}+\xi_{n-1}$.

Then the element

$$
\eta=\left[\xi_{1},\left[\ldots, \xi_{\eta}\right]\right] \text { in } L_{n} \otimes \mathbf{C}
$$

is nonzero and has the property that

$$
\phi_{n}(\eta)=\lambda_{1} \ldots \lambda_{n} \eta=\eta .
$$

Thus $\phi_{n}$ has an eigenvalue 1 , and therefore has an integral eigenvector $\theta$ corresponding to the eigenvalue 1 . Then $\theta$ is represented by $w \in F_{n}-F_{n+1}$ such that $\phi(w) \equiv w$ modulo $F_{n+1}$.

For example, taking $\phi$ to be the PV-automorphism described at the end of $\S 1$, both

$$
[x,[y, z]][x,[x, z]][y,[y, x]][z,[z, y]]
$$

and

$$
[x,[y, z]][x,[x, y]][x,[x, z]][y,[y, z]][z,[z, x]][z,[z, y]]
$$

are fixed modulo $F_{4}$. 
Hence, it is at least conceivable that this automorphism leaves something in $F_{3}$ fixed. My conjecture, which I cannot prove, is that the fixed subgroup of every PV-automorphism is trivial.

\section{REFERENCES}

1. J. L. Dyer and G. P. Scott, Periodic automorphisms of free groups, Comm. Algebra 3 (1975), 195-201.

2. W. Jaco and P. B. Shalen, Surface homeomorphisms and periodicity, Topology 16 (1977), 347-367.

3. J. W. S. Cassels, An introduction to diophantine approximation, Cambridge Univ. Press, New York, 1957.

4. N. Jacobson, Basic algebra. I, Freeman, San Francisco, Calif., 1974.

Department of Mathematics, University of California, Berkeley, California 94720 